\title{
NDT evaluation of long-term bond durability of CFRP-structural systems applied to RC highway bridges
}

\author{
Kenneth C. Crawford ${ }^{1}$
}

Received: 28 July 2015/Accepted: 1 March 2016/Published online: 16 March 2016

(c) The Author(s) 2016. This article is published with open access at Springerlink.com

\begin{abstract}
The long-term durability of CFRP structural systems applied to reinforced-concrete (RC) highway bridges is a function of the system bond behavior over time. The sustained structural load performance of strengthened bridges depends on the carbon fiber-reinforced polymer (CFRP) laminates remaining $100 \%$ bonded to concrete bridge members. Periodic testing of the CFRP-concrete bond condition is necessary to sustain load performance. The objective of this paper is to present a non-destructive testing (NDT) method designed to evaluate the bond condition and long-term durability of CFRP laminate (plate) systems applied to RC highway bridges. Using the impact-echo principle, a mobile mechanical device using light impact hammers moving along the length of a bonded CFRP plate produces unique acoustic frequencies which are a function of existing CFRP plate-concrete bond conditions. The purpose of this method is to test and locate CFRP plates de-bonded from bridge structural members to identify associated deterioration in bridge load performance. Laboratory tests of this NDT device on a CFRP plate bonded to concrete with staged voids (de-laminations) produced different frequencies for bonded and de-bonded areas of the plate. The spectra (bands) of frequencies obtained in these tests show a correlation to the CFRP-concrete bond condition and identify bonded and de-bonded areas of the plate. The results of these tests indicate that this NDT impact machine, with design improvements, can potentially provide bridge engineers a means to rapidly evaluate long
\end{abstract}

Kenneth C. Crawford

ken.crawford69@wabash.edu

1 Crawford Technologies and Applications LLC, Crawfordsville, IN 47933, USA lengths of CFRP laminates applied to multiple highway bridges within a national transportation infrastructure.

Keywords CFRP plates - Non-destructive testing · Bond · Impact-echo $\cdot$ Frequency $\cdot$ Bridges

\section{Introduction}

The load performance of CFRP-strengthened RC highway bridges is a function of the bond-interface behavior in the CFRP laminate-epoxy-concrete structural system. It is necessary to periodically evaluate the bond condition of the CFRP laminates applied to bridges to monitor potential changes (deteriorations) in bridge load performance. For transportation infrastructure owners, and in particular owners of highway bridges strengthened with CFRP systems, one primary concern is the long-term durability of the CFRP systems and sustainment of the designed bridge load capacity. Bridge owners are faced with two primary questions: (1) how do CFRP structural systems on RC highway bridges perform over extended time (15+ years) under the influence of frequent cyclic loading (heavy truck traffic), moisture, and freeze-thaw cycles? (2) How can accurate field data be obtained on CFRP-plate bond condition to evaluate changes in load performance and to establish cost-effective maintenance procedures? This concern is magnified when a national highway system has a large number of strengthened bridges and the load performance of the bridges is critically dependent on the CFRP plate-concrete bond performance, i.e., absence of debonding. CFRP plates with de-laminations can potentially produce deterioration in bridge performance (Shih et al. 2003). The NDT concept presented in this paper is one method for rapidly evaluating the bond condition of CFRP 
plates applied to multiple bridges in a national highway system. A comprehensive CFRP-strengthened bridge inspection and maintenance program with periodic testing and evaluation of the CFRP plate bond condition is necessary to support and sustain long-term bridge performance.

\section{Background}

The Republic of Macedonia carried out a NATO-funded program in 2001 to strengthen 19 highway bridges on the M2 and M1 highways on European Corridor 8 connecting Albania to Bulgaria through northeastern Macedonia. CFRP-plate structural systems were applied to multiple bridges on the $\mathrm{M} 2$, and two bridges on the M1, to increase bridge load capacity for heavy military vehicle traffic. This FRP application significantly increased the flexural bending moment of bridge structural members. A total of $14,600 \mathrm{~m}$ of CFRP plates were applied to the 19 bridges having a bonded area of 1318 square meters (Crawford and Nikolovski 2007). Consequently, the condition of CFRP laminate-concrete bond becomes a critical element in sustaining long-term bridge load performance. After 15 years the condition of the CFRP-concrete bond on the 19 bridges is an open question: are the CFRP plates still $100 \%$ bonded to the bridge structural members? Is the current bridge load performance sustaining the original designed CFRP-strengthened flexural bending moment? The challenge is having an effective field testing process to evaluate the CFRP-plate bond condition to determine if any changes in bridge performance have occurred. Since 2006 an additional 82 bridges in Macedonia have been strengthened and refurbished with FRP systems in varying applications. 46 bridges in the Ukraine, nine bridges in Montenegro, and six bridges in Kosovo have also been refurbished and strengthened. The most recent contract in Macedonia was awarded in December 2013, to repair and strengthen 16 bridges on the M1 (European Corridor 10) between Skopje and $50 \mathrm{~km}$ south to Veles. To sustain the designed load performance on this large number of CFRPstrengthened bridges, a reliable field testing procedure in bridge inspection and maintenance is needed to periodically verify bond condition and associated bridge load performance.

\section{Scope of strengthened-bridge applications requiring CFRP-bond testing}

The 19 bridges described above have varying degrees of CFRP laminations applied to the structural members of different types of slab and girder bridges. Figures 1 and 2

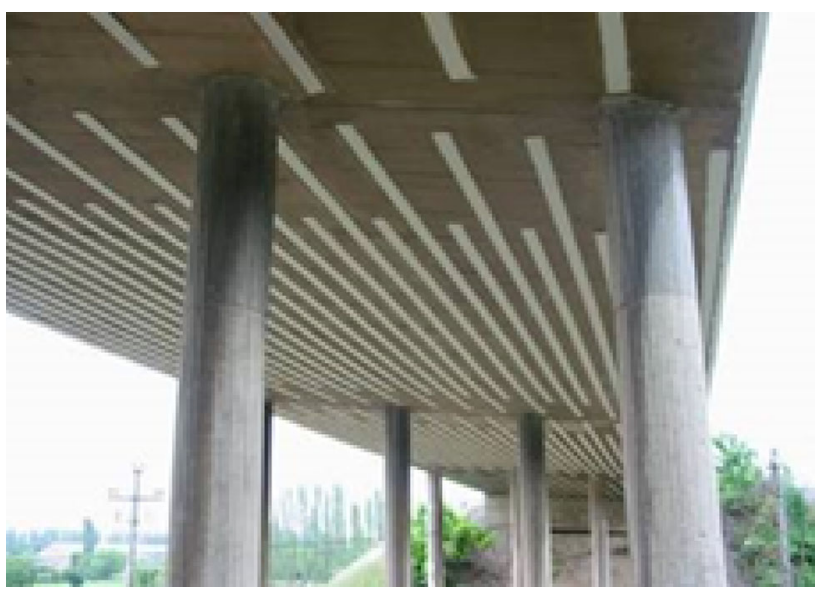

Fig. 1 Bridge B2-N on M1-938 m

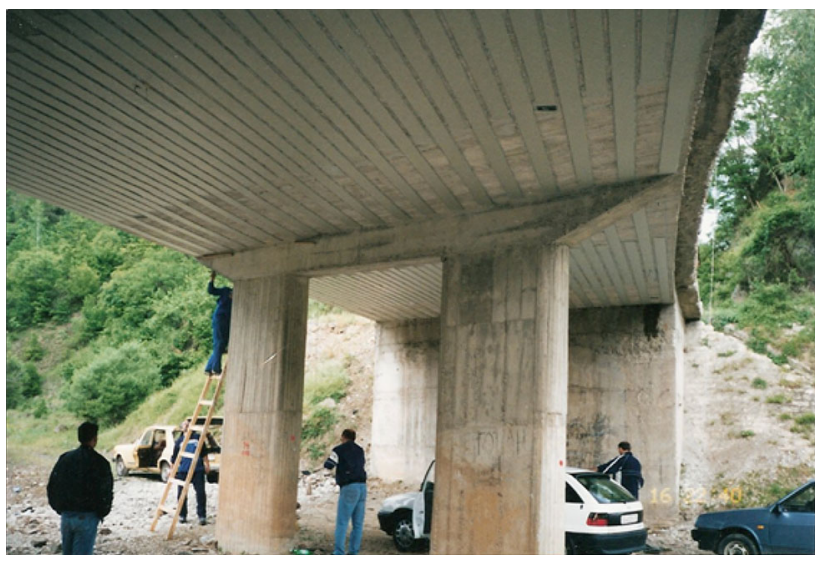

Fig. 2 Bridge B36 on M2-1032 m

illustrate the lengths of CFRP plates applied to two slab bridges, each having about $1 \mathrm{~km}$ of CFRP material. Table 1 describes, as an example, the length and area of CFRP plate material applied on ten of the 19 bridges.

One example of the degree of CFRP strengthening applied to the 19 slab and girder bridges on the M1 and M2 is illustrated by the bridge B2-N, Fig. 1, at km marker $10+7$ near Kumanovo on the M1. B2-N is a $46 \mathrm{~m}$ bridge in three spans, $10.2 \mathrm{~m}$ wide, $66 \mathrm{~cm}$ thick, constructed in 1962 in accordance with YU Regulations PTP-5 for an M-25 vehicle. For this bridge the ultimate bending moment before strengthening was $399.8 \mathrm{kNm}$. The required ultimate bending moment after strengthening was $659.2 \mathrm{kNm}$, a $65 \%$ increase in the bending moment. $938 \mathrm{~m}$ of CFRP plates were applied to the bridge to achieve the $659 \mathrm{kNm}$ bending moment (Crawford 2008). To sustain this bending moment $100 \%$ of the plates must be bonded to the bridge. It therefore becomes necessary to periodically evaluate the condition of the laminate bond to verify the designed load performance is still being sustained by the applied CFRP system. 
Table 1 Length/area of applied CFRP plates on ten M2 and M1 bridges

\begin{tabular}{|c|c|c|c|c|c|c|c|}
\hline \multicolumn{5}{|c|}{ Selected CFRP-strengthened bridges on the M2 and M1 } & \multicolumn{3}{|c|}{ Total CFRP plate length $(\mathrm{m})$ and area $\left(\mathrm{m}^{2}\right)$} \\
\hline Bridge no. & Location on M2/M1 & Bridge type & Bridge length (m) & No. spans & No. plates & Plate length & Plate area \\
\hline B7 & $14+027$ & Girder & 120 & 6 & 72 & 1478 & 116.1 \\
\hline B11 & $21+876$ & Slab & 10 & 1 & 26 & 198 & 29.6 \\
\hline $\mathrm{B} 18$ & $38+444$ & Slab & 36 & 1 & 54 & 218 & 32.6 \\
\hline B22 & $41+786$ & Slab & 30 & 1 & 60 & 1308 & 196.3 \\
\hline B28 & $49+631$ & Girder & 50 & 3 & 60 & 1210 & 100.6 \\
\hline B35 & $66+058$ & Girder & 52 & 3 & 20 & 346 & 41.2 \\
\hline B36 & $67+409$ & Slab & 21 & 2 & 80 & 1032 & 82.8 \\
\hline B37 & $68+452$ & Girder & 17 & 1 & 36 & 415 & 34.4 \\
\hline B39 & $71+211$ & Girder & 85 & 4 & 48 & 1778 & 146.2 \\
\hline $\mathrm{B} 2-\mathrm{N}$ & $10+07 \mathrm{M} 1$ & Slab & 46 & 4 & 96 & 938 & 112.5 \\
\hline
\end{tabular}

\section{Significance of research}

Owners using CFRP-structural systems on large numbers of bridges within a national transportation system have an ongoing requirement to evaluate the bond condition of FRP composites applied to RC highway bridges in order to monitor long-term bridge load performance. While the impact-echo method has been effective in testing concrete structures for many years, its application for evaluating CFRP-structural systems applied to bridges is not fully developed and used in the field. The development of the mobile impact-echo machine presented in this paper expands the application of NDT procedures to evaluate bridge CFRP plate-concrete bond conditions in the field.

\section{Impact-echo NDT evaluation of CFRP-concrete bond}

Among available methods of non-destructive testing (NDT) to evaluate the condition of FRP material bonded to concrete structures, among which are thermal imaging, high-frequency radiation, and laser vibrometry, the use of the impact-echo method presented provides an effective and low-cost means of rapidly assessing the bond condition of the CFRP laminate-epoxy-concrete tri-layered structure applied to large numbers of strengthened bridges. The advantage of this method over the three methods above is ease and speed of testing long lengths of CFRP plates.

\section{Impact-echo principle}

The concept of impact-echo testing, invented by the US National Bureau of Standards (NBS) in the mid-1980s, applies acoustic methods for non-destructive evaluation of concrete and masonry structures. The principle of the impact-echo method involves transmission of energy of a mechanical impact from a small steel sphere into the mass of a structure to generate transient sinusoidal stress (sound) waves. Low-frequency impact stress waves propagating into a structure are reflected by internal surfaces and flaws (Sansalone and Streett 1998). The patterns present in the reflected waveforms and spectra provide information about the existence and locations of structural flaws, e.g., debonding. Multiple reflections of stress waves between the impact surface and flaws give rise to transient resonances, which can be identified in the frequency spectrum, and used to evaluate the integrity of the structure. The resulting wave displacement signals in the time domain are transformed into the frequency domain in which plots of amplitude versus frequency (spectra) are obtained. Surface displacements caused by these wave reflections are recorded by a transducer located adjacent to the point of impact. Impact-echo tests on concrete structures, such as CFRP plates applied to bridge structural members, produce distinctive waveforms and frequencies, in which dominant patterns in the number and distribution of peaks in the spectra are easily identified. Voids and de-laminations (flaws) in the CFRP-concrete structure change impact waveform patterns (frequency spectra) and provide qualitative and quantitative information about the existence and location of the structural deficiencies (Ray et al. 2007).

The quality of impact-echo testing and the data obtained from the CFRP-concrete structure is a function of the impact hammer configuration and the sinusoidal waveform it provides. The objective is to achieve maximum waveform resolution with optimized hammer sphere size and impact force. In a mechanical system the impact of a small steel sphere can be represented as a unit impulse function with impact force $g(t)$. The impact energy $I(\tau)$, Eq. 1 , is the integral of the impact force $g(t)$ over the impulse time 
interval $\left(t_{1}-t_{0}\right)$. If force $g(t)=c$ and $t_{0}=0$ with $t_{1}<180 \mu$ s such that $0<\tau<180 \mu$ s, the integral of $I(\tau)$ from $t_{0}$ to $t_{1}$ becomes the total impact energy.

$I(\tau)=\int_{t_{0}}^{t_{1}} g(t) \mathrm{d} t=2 \tau c=1$

The unit impulse is the total hammer impact energy transmitted into the CFRP-concrete bonded structure. The input energy $I(\tau)=c=1 / 2\left(t_{1}-t_{0}\right)$. For the NDT device used in these tests, the force, where $g(t)=c$, of each hammer impact on the surface of the CFRP plate, was equal to $5 \mathrm{~N}$ (about $1 \mathrm{lb}$ ). The short duration of a $5-\mathrm{N}$ impact force is sufficient for detecting flaws (de-bonding) in CFRP plate-concrete bond structure.

\section{Fourier transform of impact signal}

The impulse signal generated by the hammer impact on the surface of the CFRP plate produces a complex sinusoidal waveform in the time domain with a unique frequency that has amplitude and phase. The objective of the test is to identify the waveform frequency of the impact and to determine how the frequency of the impacts changes over the length of the CFRP plate. Given two sinusoidal waveforms $t_{1}$ and $t_{2}$, each $t$ represents a different time and position on the plate in the hammer impact sequence. If $t_{1}$ is the signal of a bonded plate and $t_{2}$ is that of a de-bonded plate, each sinusoidal waveform signal $t$ will have a different time phase and unique frequency. The equation for the sinusoidal signal $t$ in the time domain is $f(t)=\cos \omega t+i \sin \omega t$, where $\omega=2 \pi f$ is the angular frequency in radians/sec. Using the Fourier transform in Eq. 2 the frequency $F(\omega)$ of the sinusoidal signal $f(t)$ is found by integrating $f(t)$ in its exponential form $e^{-\mathrm{i} \omega \mathrm{t}}$ (Euler's formula) from $T_{1}$ to $T_{2}$.

$F(\omega)=\int_{T_{1}}^{T_{2}} f(t) e^{-i \omega t} \mathrm{~d}(t)$

If $f(t)$ from $T_{1}$ to $T_{2}$ is the duration of impulse generated in the time domain then the time signals $t_{1}$ and $t_{2}$ in the Fourier transform become the frequency signals $f_{1}$ to $f_{2}$. If $f_{1}$ and $f_{2}$ are two different frequencies such that if $f_{1}<f_{2}$, then the impact signals $t_{1}$ and $t_{2}$ indicate two different bond conditions, e.g., de-bonded versus bonded CFRP plates. With the Fourier transform providing the frequencies of the time signals, changes in frequencies of the impact signals as the NDT device moves over the CFRP plate can be identified. If the impact frequencies across the CFRP plate remain constant, the plate is fully bonded. Any changes to lower frequencies can indicate some plate de-bonding has occurred and may be present over the length of the CFRP plate.

\section{Mobile impact-echo machine}

The NDT impact machine, Fig. 3, is a four-wheel mobile device consisting of two spring-activated levers mounted $5 \mathrm{~cm}$ apart in an enclosed frame. One actuator wheel alternately lifts each lever $10 \mathrm{~mm}$ to drop an impact bolt on the CFRP plate surface. Each lever is driven by an adjustable-tensioned band (spring) to obtain a consistent $5 \mathrm{~N}$ impact force along the length of the plate. The impacts occur alternately along two parallel tracks on the plate (Crawford 2011). The impact impulse energy is produced by a small steel sphere (head of a carriage bolt) mounted on the oscillating lever. The size of the sphere is optimized to produce maximum input energy into the CFRP plate surface. The resin matrix of the CFRP plate is a softer material than the concrete and does not produce as sharp a stress wave (sound) as an impact on a concrete surface. This means the system for detection and recording the impact signal must be more sensitive (responsive) to detect changes in frequency on the CFRP plate as bond conditions with the concrete change. Frequency differences for bonded versus de-bonded areas are generally smaller for changes in CFRP-plate bonding than for flaws in solid concrete structures.

\section{CFRP test plate}

To test the impact machine, a $10 \mathrm{~cm}$ by $3.0 \mathrm{~m}$ Sika CarboDur S1012 rigid plate, $1.2 \mathrm{~mm}$ thick, Fig. 4 , was fixed with double-backed adhesive tape (in place of epoxy) on a level concrete surface. The bonding adhesive was spaced to create $10-40 \mathrm{~cm}$ delaminated (de-bonded) areas between the plate and the concrete. In Fig. 4 the de-bonded areas are marked in gray on the plate surface, showing their relationship to the bonded areas along the length of the test plate. The frequencies generated by the multiple impacts on the CFRP test plate, Fig. 4, are shown on two horizontal lines, each with sets of vertical lines. Each vertical line is a single impact point with a unique frequency indicating a bonded plate on the $3.26 \mathrm{kHz}$ line and a de-bonded area of the plate on the $2.88 \mathrm{kHz}$ line.

\section{Testing protocol}

The objective of this testing protocol is to verify the concept of applying the NDT impact-echo technique to identify de-bonding of the epoxy-bond structure between CFRP laminates and concrete bridge structural members. The goal is to determine the feasibility of using this testing procedure in the field on CFRP-strengthened bridges. The testing protocol was conducted in two phases: (1) 
Fig. 3 Mobile impact machine with dual light impact hammers
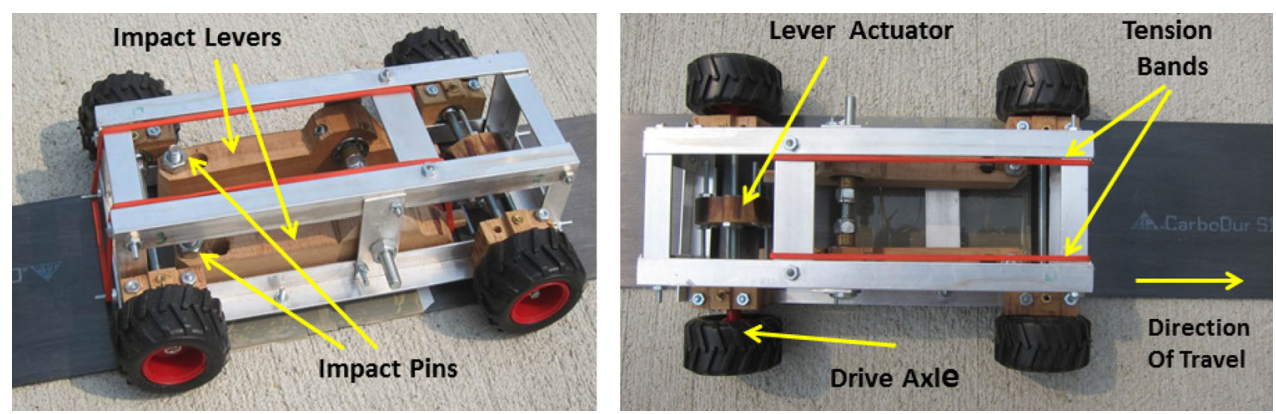

Fig. 4 CFRP test plate with point-of-impact frequencies

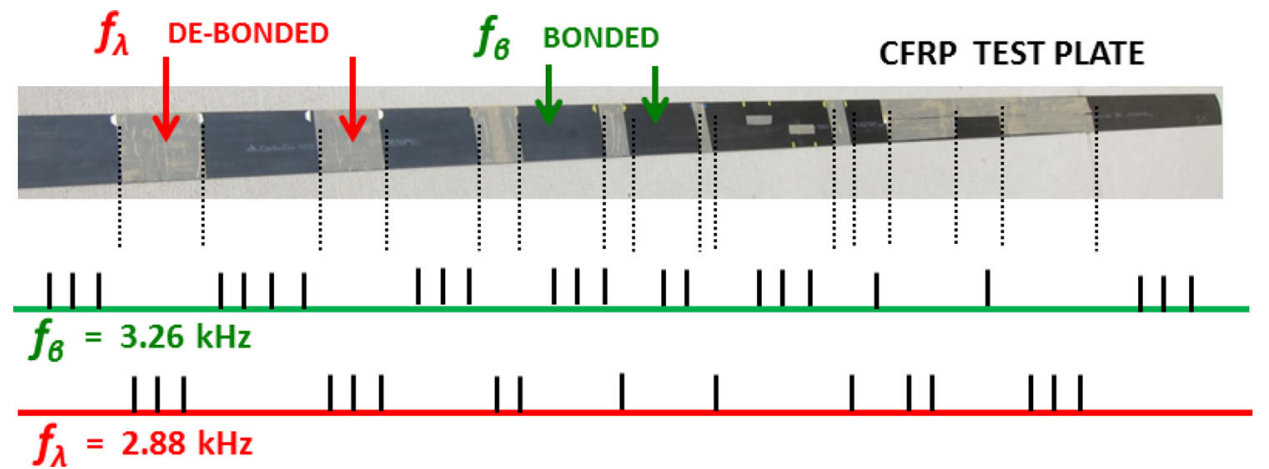

performing the impact-echo evaluation on the CFRP test plate and recording the resulting acoustic frequencies from the impacts. (2) Analyzing the recorded time-domain signals on the oscilloscope for pulse waveform structure and on the spectrum analyzer in the frequency domain for frequency variations and frequency components of the impact signals.

\section{Test procedure}

The mobile impact-echo machine shown in Fig. 3 was moved (rolled) along the length of the CFRP test plate, Fig. 4, at a constant speed. The two impact hammer levers were lifted alternately by an actuator wheel. With each drop of the lever the impact pin produced an impact stress wave (acoustic sound) every $3 \mathrm{~cm}$, with the machine producing 33 impacts per meter across the surface of the CFRP plate. Figure 5 is a schematic of the mobile device producing multiple impact stress waves with successive impacts on the CFRP plate bonded to the concrete bridge structural member. Each impact produces a single acoustic signal with a unique frequency. The frequencies produced are a function of the bond condition at that specific point on the CFRP plate.

Impact 1 on a bonded plate in Fig. 5 produces a stress wave with frequency $f_{\beta}$. Impact 2 on a de-bonded area of the plate produces a stress wave with a lower frequency $f_{\lambda}$. Multiple runs with the impact device were conducted over the length of the CFRP test plate. The impact signals were received through a transducer (microphone) mounted on the mobile device and recorded on a broad-band receiver for frequency spectrum analysis.

\section{Test results}

With the impact machine producing a series of 99 impacts along the length of the $3.0 \mathrm{~m}$ CFRP test plate the change in frequencies between the two CFRP-bond states (bonded vs. de-bonded) was distinct and measurable. This means the varying bond conditions of the CFRP plate did produce different impact frequencies. Test results with successive impacts on the CFRP test plate indicated voids (plate delaminations) were present when $f_{\lambda}<f_{\beta}$. The recorded impact signals were fed into a Tektronix $2712(1 \mathrm{kHz}$ to $1.86 \mathrm{GHz})$ spectrum analyser to display the range of frequencies produced by impacts over the bonded and debonded areas of the CFRP test plate. To do this the recorded signals were connected into the analyser preamplifier using a 2-kHz radio frequency (RF) center frequency $\boldsymbol{F}_{\mathbf{c}}$ providing a reference marker for the input signals. The analyser ten-division horizontal scan was set to $1 \mathrm{kHz} / \mathrm{di}$ vision to provide a $10 \mathrm{kHz}$ scanning span for the input frequency spectrum. A bandwidth resolution of $300 \mathrm{kHz}$ enabled the recorded impact signal waveforms to be displayed on the spectrum analyser as smaller envelopes right of the $\boldsymbol{F}_{\mathbf{c}}$ center frequency. The frequency spectra of the 
Fig. 5 Schematic of stress waves produced by impact machine
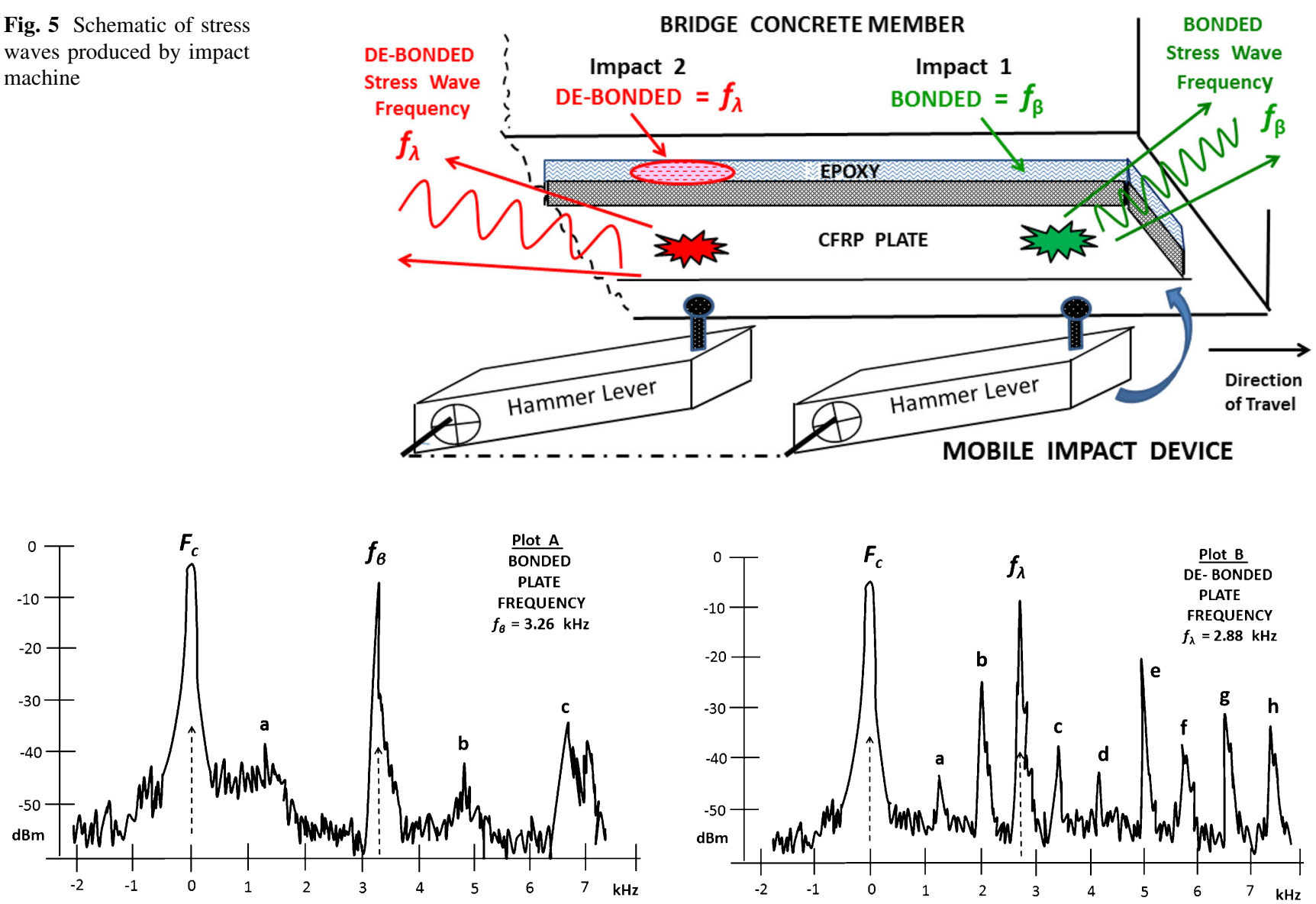

Fig. 6 Frequency spectrum display of bonded and de-bonded frequencies

impact signals are shown in plots A and B in Fig. 6. Both the bonded frequencies with primary peaks of $3.26 \mathrm{kHz}$ and the de-bonded frequencies with primary peaks of $2.88 \mathrm{kHz}$ appeared as clear and distinct frequencies with a measurable separation from the center frequency $\boldsymbol{F}_{\mathbf{c}}$ in the range from 2.5 to $3.5 \mathrm{kHz}$. In these tests the amplitudes of the bonded versus de-bonded signals differed by -5 to $-10 \mathrm{dBm}$. Smaller signal peaks right of center frequency $\boldsymbol{F}_{\mathbf{c}}$ are positive harmonics of the impact frequencies. The harmonics shown on the analyser as peaks a through $\mathbf{g}$, Fig. 6, are frequency components of the impact signals.

For this impact sequence the spectrum analyser indicated the frequency $f_{\beta}$ for bonded CFRP plate was $3.26 \mathrm{kHz}$, and the frequencies for de-bonded areas of the CFRP plate fell in a narrow frequency range around $2.88 \mathrm{kHz}$, Fig. 6. The impact frequencies $f_{\lambda}$ for the debonded areas were 10-12\% lower than the impact frequencies $f_{\beta}$ for bonded areas. It was noted the range of frequencies $f_{\lambda}$ over all de-bonded areas was consistent and remained in range of $10 \%$ below the bonded frequencies. As the voids under the test plate became smaller $(<10 \mathrm{~cm})$ the frequency difference between $f_{\lambda}$ and $f_{\beta}$ became more difficult to detect, possibly a function of the sensitivity of the recording system used in the tests. It was observed the frequencies for both the bonded and de-bonded areas varied slightly for each impact but remained generally within a $15 \%$ range of the primary (strongest) signal frequency.

In Fig. 7 two separate frequency spectra bands are plotted for each set of recorded bonded and de-bonded

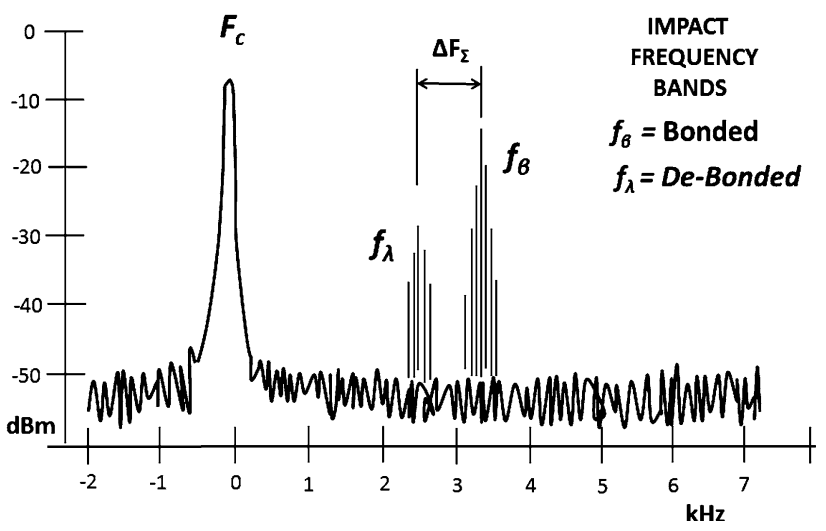

Fig. 7 Frequency bands for bonded and de-bonded impact signals 

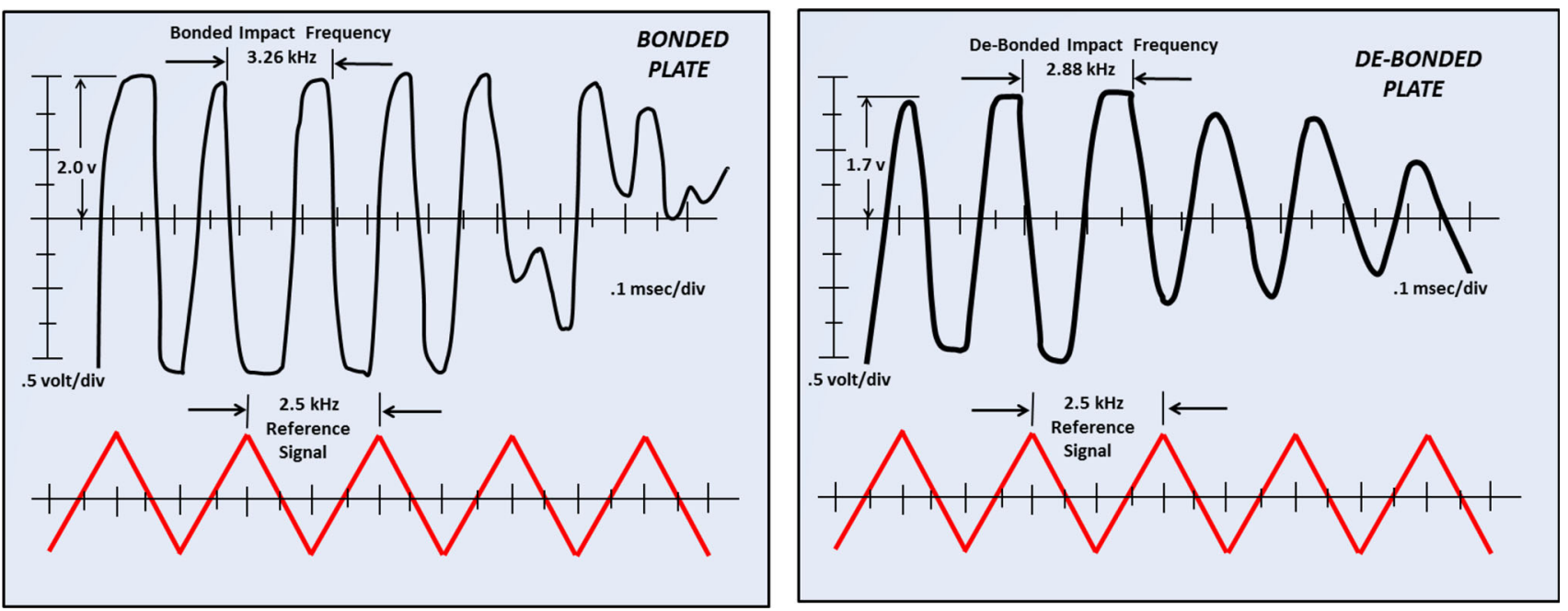

Fig. 8 Oscilloscope plots of bonded and de-bonded impact signal waveforms

impact signal frequencies. The majority of the bonded impact frequencies fell into a narrow frequency range between 3.10 and $3.40 \mathrm{kHz}$, indicated with frequency band $f_{\beta}$. The de-bonded frequencies also fell within a narrow frequency range between 2.6 and $2.9 \mathrm{kHz}$, shown by frequency band $f_{\lambda}$. The separation between the bonded and debonded frequency bands averaged approximately $300 \mathrm{~Hz}$, indicated by $\Delta \mathbf{F}_{\boldsymbol{\Sigma}}$ in Fig. 7.

The recorded signals were also displayed on a Tektronix 475 dual-trace oscilloscope to analyze the waveform and structure of the impact signals. The input signals were displayed on the top trace of the oscilloscope, plotted in Fig. 8 , with a $2.5-\mathrm{kHz}$ sawtooth signal on the bottom trace providing a frequency reference scale. Both bonded and debonded impact signals had clear sinusoidal waveforms resembling more irregular square waves than pure sine waves. Both bonded and de-bonded waveforms remained consistent for successive impacts across the length of the CFRP test plate.

\section{Discussion}

The difference between bonded frequency $\boldsymbol{f}_{\boldsymbol{\beta}}$ and de-bonded frequency $f_{\lambda}$ is represented by $\Delta \mathbf{F}_{\Sigma}$, Fig. 7 . The frequency difference $\Delta \mathbf{F}_{\boldsymbol{\Sigma}}$ is a function of the condition of the CFRPconcrete bond and expresses the degree of de-bonding of the CFRP plate over the length of the plate. When $\Delta \mathbf{F}_{\mathbf{\Sigma}}=0$, then de-bonded frequencies $f_{\lambda}$ are not present, meaning the CFRP plate is fully bonded. If $\Delta \mathbf{F}_{\boldsymbol{\Sigma}}>0.3 \mathrm{kHz}$ then some debonding of the CFRP plate may have occurred. The degree of de-bonding can be quantified in terms of number of debonded frequencies occurring per meter over the length of the CFRP plate. This is accomplished by counting the number of impacts per meter producing $f_{\lambda}$ frequencies using a distance timing signal on the NDT device. The number of timing signals from the starting point will determine the location of the de-bonded areas on the plate. As the number of $f_{\lambda}$ frequencies are recorded with their locations an accurate diagram of the de-bonded areas of the CFRP plate can be plotted and quantified. The data produced with this impactecho method provide the bridge engineer an extensive picture of the CFRP plate bond condition. As bond condition evaluation tests are performed in periodic bridge maintenance protocols, the long-term performance and bond durability of the CFRP-structural systems applied to RC highway bridges can be determined, providing data to more accurately plan and program bridge maintenance costs.

\section{Conclusion}

The following points can be concluded from the tests presented in this paper:

1. Use of the NDT impact-echo method presented in this paper may be effective in evaluating CFRP laminate bond condition on RC bridge structural members.

2. Laboratory tests of the impact-echo machine with associated signal analysis show a correlation between signal frequency and CFRP-laminate bond condition.

3. The impact-echo method presented may be an effective means for rapidly evaluating the bond condition of long lengths of CFRP plates applied to large numbers of highway bridges.

4. Much research is needed on developing methods for signal processing and analysis of CFRP-laminate impact data points. 
5. The impact tests presented in this paper indicate the impact-echo method can potentially identify changes in bridge load performance resulting from de-bonded CFRP laminate systems.

To monitor load performance of RC highway bridges strengthened with CFRP-structural systems, there is a growing need for effective field procedures to evaluate the bond structural condition of CFRP plates applied to extensive numbers of highway bridges. The NDT device presented in this paper using the technology of impact-echo testing, with further development, can have direct application to current bridge testing and maintenance programs for owners of CFRP-strengthened bridges. This NDT method can potentially provide bridge engineers a practical procedure to evaluate large areas of CFRP plates applied to multiple highway bridges in a national highway.

Acknowledgments The author thanks Wabash College for their support in the research for this paper.

\section{Compliance with ethical standards}

Conflict of interest The author declares he has no competing or conflicts of interest.

Open Access This article is distributed under the terms of the Creative Commons Attribution 4.0 International License (http:// creativecommons.org/licenses/by/4.0/), which permits unrestricted use, distribution, and reproduction in any medium, provided you give appropriate credit to the original author(s) and the source, provide a link to the Creative Commons license, and indicate if changes were made.

\section{References}

Crawford K (2008) Measuring performance of concrete bridges strengthened with FRP-structural systems. Advanced composite materials in bridges and structures (ACMBS-V), paper 53

Crawford K (2011) Non-destructive testing of FRP-structural systems applied to concrete bridges, international symposium on nondestructive testing of materials and structures, paper 102

Crawford K, Nikolovski T (2007) The application of ACI 440 for FRP system design to strengthen 19 concrete highway bridges on European corridor 8". In: 8th international conference on fiber reinforced polymer for reinforcement of concrete structures, paper $15-4$, pp 570-571

Ray BC, Clegg DW, Hasan ST (2007) Evaluation of defects in FRP composites by NDT techniques. J Reinf Plast Compos 26:1187-1192

Sansalone M, Streett W (1998) The impact-echo method. NDTnet $3(2): 3-7$

Shih J, Tann D, Hu C, Delpak R, Andreou E (2003) Remote sensing of air blisters in concrete-FRP bond layer using IR thermography. Int J Mater Prod Technol 19:174-187 